\title{
Lanthanide-doped metal-organic frameworks with multicolor mechanoluminescence
}

\author{
Wenwei Chen ${ }^{1,2}$, Yixi Zhuang ${ }^{1,2^{*}}$, Changjian Chen $^{1,2}$, Ying Lv ${ }^{1,2}$, Ming-Sheng Wang ${ }^{1}$ and \\ Rong-Jun $\mathrm{Xie}^{1,2^{*}}$
}

\begin{abstract}
Metal-organic frameworks (MOFs) and mechanoluminescent (ML) materials have been considered as two types of promising materials that have their own application fields. It would be amazing to endow one material with the advantages of ML and MOFs, thus broadening their applications. However, there are quite few investigations on this topic, and the ML mechanism in ML-MOFs remains unclear. In this study, we proposed a strategy for developing ML-MOFs by doping lanthanide ions into the non-centrosymmetric SBD $\left([\operatorname{Sr}(\mu-B D C)(D M F)]_{\infty}\right)$ MOF, and successfully synthesized a series of lanthanide-doped MOFs Ln-SBD $(\mathrm{Ln}=\mathrm{Tb}, \mathrm{Dy}, \mathrm{Sm}$, Eu) and $\mathrm{Tb}_{1-x} \mathrm{Eu}_{x}$-SBD $(x=0.2,0.4,0.6,0.8)$ with multicolor ML. The lanthanide ions were uniformly distributed in the matrix of the SBD-MOF, and occupied the Sr site. The MLMOFs exhibited intense multicolor ML emissions varying from green to yellow to red by changing the co-doping ratios and species of lanthanide ions. The similar ML and photoluminescence (PL) spectra indicated that the ML emission was assigned to the radiative transition from the excited states to the ground states of lanthanide ions. The radiative transition was induced by the electron bombardment process that originated from the piezoelectric effect of the non-centrosymmetric SBD host. In addition, a pioneering temperature sensing research based on ML was carried out, which is promising for realizing dual-functional detection of stress and temperature without excitation light sources. This study gives a unique insight for developing more versatile and interesting smart materials by combining the versatility of MOF with the ML emission, imparting additional values to both MOF and ML materials. Moreover, this study provides a general rule for selecting MOFs with an acentric structure as the host for ML materials.
\end{abstract}

Keywords: metal-organic frameworks, lanthanide-doped, multicolor mechanoluminescence, temperature sensing

\section{INTRODUCTION}

Metal-organic frameworks (MOFs), which are a kind of organic-inorganic hybrid porous materials self-assembled from metal ions or clusters with organic ligands and are also known as porous coordination polymers (CPs), have attracted striking attention in the past decades owing to their potential applications in adsorption, separation, storage, catalysis, delivery and sensing [1-18]. Recently, a special subclass of MOFs, i.e., luminescent MOFs, has emerged and been considered as very promising multifunctional luminescent materials for diverse applications, such as luminescent sensing, photocatalysis, anticounterfeiting, laser, and white light emitting [11-27]. Although approximately one-tenth of MOFs-related articles focus on the topic of luminescence, nearly all of those papers on luminescent MOFs address the photoluminescence (PL) phenomena. To the best of our knowledge, there are very few investigations on the mechanoluminescence (ML) of MOFs [28-32].

The so-called ML refers to the light emission of solidstate materials under mechanical stimulus, which was first recorded by Francis Bacon [33] in The Advancement of Learning and has drawn considerable attention due to its potential applications in stress monitoring, E-signature systems, artificial skins, and optical sensors [34-41]. Generally, ML can be further subdivided into three subcategories, including elastic ML (EML), plastic ML (PML) and fracto ML (FML), corresponding to ML induced by elastic deformation, plastic deformation and fracture of solids, respectively [42-44]. Till now, thousands of ML materials have been reported, and some typical ML materials, such as $\mathrm{ZnS}: \mathrm{Mn}^{2+}, \mathrm{SrAl}_{2} \mathrm{O}_{4}: \mathrm{Eu}^{2+}, \mathrm{SrZnOS}: \mathrm{Ln}^{3+}$, sugar, impure saccharin, phenanthrene, coumarin, ditriphenylphosphine oxide manganese bromide and

\footnotetext{
${ }^{1}$ College of Materials, Xiamen University, Xiamen 361005, China

${ }^{2}$ Fujian Provincial Key Laboratory of Materials Genome, Xiamen University, Xiamen 361005, China

* Corresponding authors (emails: zhuangyixi@xmu.edu.cn (Zhuang Y); rjxie@xmu.edu.cn (Xie RJ))
} 
europium tetrakis (dibenzoylmethide) triethyl ammonium, have shown intense ML emission [41-44]. These investigations concentrated much on the ML intensity and spectra of materials, but less on adding values to their ML properties, which is thus hard to broaden their applications. It would be a choice to develop ML-MOFs by adding ML emission to the versatility of MOFs or adding MOF structures to ML materials, achieving hybrid functions in ML-MOFs. For example, ML-MOFs with the gas detection function could simultaneously detect stress and gas, while ML-MOFs with the temperature sensing function could simultaneously monitor stress and temperature. However, it remains a challenging to develop ML-MOFs.

As far as we know, metal-organic complexes with ML properties have emerged as early as the 1960s [45] and have been extensively investigated as well [46-51], but similar studies on CPs, especially MOFs, have not been reported until 2004 [28]. Thereafter, Eliseeva et al. [29] obtained several ML-MOFs $\left[\operatorname{Ln}(\mathrm{hfa})_{3}(\mathrm{Q})\right]_{\infty},(\mathrm{Ln}=\mathrm{Eu}$, $\mathrm{Tb}$; hfa = hexafluoroacetylacetonate; $\mathrm{Q}=1$,4-diacetoxybenzene (ACETBZ), 1,4-dimethyltherephtalate $(\mathrm{DMTPH})$ ) by assembling lanthanide $\beta$-diketonates and aromatic bidentate $\mathrm{O}$-donor ligands together. The highly efficient ML detectable to the naked eyes was ascribed to the near-unity efficiency of light emission from the excited state generated upon fracture of the material. Hasegawa's group [30] investigated another brilliant ML-MOF $\left[\mathrm{Eu}(\mathrm{hfa})_{3}(\mathrm{BIPYPO})\right]_{\infty}$, which is composed of luminescent Eu complexes with hfa and bidentate phosphane oxide ligands 3,3'-bis(diphenylphosphoryl)-2,2'-bipyridine (BIPYPO). They believed that the effective ML was caused by the non-centrosymmetric space group $C c$ and the low-vibrationalfrequency hfa and phosphane oxide ligands. The same authors also reported a europium ML-MOF $\left[\mathrm{Eu}(\mathrm{hfa})_{3}\right.$ $\left.(\mathrm{TPPB})_{2}\right]_{n}$ based on $\left[\operatorname{Ln}(\mathrm{hfa})_{3}\right]$ units and rigid triangular spacer ligands tris(4-diphenylphosphorylphenyl)benzene (TPPB) [31]. Recently, they synthesized a series of ML-MOFs $\left[\operatorname{Ln}(\mathrm{hfa})_{3} \mathrm{Q}\right]_{n} \quad(\mathrm{Q}=2,5$-bis(diphenylphosphoryl)furan (DPF), 2,5-bis(diphenylphosphoryl)thiophene (DPT), 3,4-bis(diphenylphosphoryl) ethylenedioxythiophene (DPEDOT); $\mathrm{Ln}=\mathrm{Eu}, \mathrm{Tb}$, or the mixture thereof) with high brightness by introducing polar bridging ligands such as furan groups [32]. The relationship between ML and PL was clearly demonstrated by the ML and PL spectra of Tb/Eu mixed CPs. The results indicated that there were discrete excitation mechanisms for ML and PL phenomena. It is worth noting that the most aforementioned ML-MOFs in- cluding $\mathrm{Eu}(\mathrm{TPA})_{3}(\mathrm{HTPA})_{2}(\mathrm{TPA}=$ thiophene carboxylic acid) [28], [Ln(hfa $\left.)_{3}(\mathrm{DMTPH})\right]_{\infty}[29],\left[\mathrm{Eu}(\mathrm{hfa})_{3^{-}}\right.$ $(\mathrm{BIPYPO})]_{\infty} \quad[30], \quad\left[\operatorname{Ln}(\mathrm{hfa})_{3} \mathrm{DPF}\right]_{n}$ and $\left[\operatorname{Ln}(\mathrm{hfa})_{3^{-}}\right.$ $\mathrm{DPEDOT}_{n}[32]$ possess acentric space groups. Statistically, ML performances of most inorganic and organic materials are concerned with a non-centrosymmetric structure. Consequently, although the correlation between ML emission and acentric structure is barely discussed in these ML-MOFs, it can be easily inferred that it is the acentric structure, or the piezoelectricity resulting from acentric structure, that facilitates the occurrence of strong ML properties. Therefore, whether the MOF materials have an acentric structure is a key principle for the design and development of novel ML-MOFs. In addition, all these reported ML-MOF materials were composed of complicated ligands and lanthanide ions, which inevitably lead to difficulties in material synthesis, cost control, rare earth resource conservation, and so on. Moreover, in comparison to the design and synthesis of materials, the underlying ML mechanisms still remain ambiguous. In addition, although there have been many studies on temperature sensing based on PL, those based on ML has not been reported yet $[15-18,52,53]$. If ML materials have temperature sensing properties, it is of great significance to realize the dual function detection of stress and temperature, and it will also greatly promote the multifunction of ML materials.

Therefore, it is a good choice to develop novel MLMOFs by screening the easily available non-lanthanidebased MOF matrices with an acentric structure and then adding luminescent lanthanide ions. In this study, an acentric alkaline-based MOF $[\operatorname{Sr}(\mu-B D C)(D M F)]_{\infty}(S B D$; where $\mathrm{BDC}$ is benzene-1,4-dicarboxylate, DMF is $\mathrm{N}, \mathrm{N}$ dimethylformamide) with good thermal stability and easy availability was chosen as the matrix [54,55], and some lanthanide ions including $\mathrm{Tb}^{3+}, \mathrm{Dy}^{3+}, \mathrm{Sm}^{3+}$ and $\mathrm{Eu}^{3+}$ were chosen as dopants for luminescence. A series of lanthanide-doped MOFs Ln-SBD ( $\mathrm{Ln}=\mathrm{Tb}, \mathrm{Dy}, \mathrm{Sm}, \mathrm{Eu})$ and $\mathrm{Tb}_{1-x} \mathrm{Eu}_{x}$-SBD $(x=0.2,0.4,0.6,0.8)$ with multicolor ML were successfully synthesized by doping $\mathrm{Ln}^{3+}$ ions into SBD MOF host. The $\mathrm{Tb}^{3+}$ ion doping does not destroy the excellent thermal stability of the SBD matrix, but also promises bright green PL in the doped sample. More interestingly, a strong green ML emission in Tb-SBD was observed. In addition, tunable spectra from green to yellow to red in these ML-MOF materials were realized by changing the co-doping ratios and species of lanthanide ions. Finally, a pioneering temperature sensing research based on ML was carried out in the obtained MLMOFs. 


\section{EXPERIMENTAL SECTION}

\section{Chemicals}

BDC and DMF were purchased from Aladdin Ltd. (Shanghai, China). Strontium nitrate $\left(\mathrm{Sr}\left(\mathrm{NO}_{3}\right)_{2}\right)$ was purchased from Xilong Scientific Co., Ltd. (Guangdong, China) and all the lanthanide nitrates, including $\mathrm{Tb}\left(\mathrm{NO}_{3}\right)_{3} \cdot 5 \mathrm{H}_{2} \mathrm{O}, \mathrm{Dy}\left(\mathrm{NO}_{3}\right)_{3} \cdot 6 \mathrm{H}_{2} \mathrm{O}, \mathrm{Sm}\left(\mathrm{NO}_{3}\right)_{3} \cdot 6 \mathrm{H}_{2} \mathrm{O}$ and $\mathrm{Eu}\left(\mathrm{NO}_{3}\right)_{3} \cdot 6 \mathrm{H}_{2} \mathrm{O}$ were purchased from Zhongnuo Advanced Material (Beijing) Technology Co., Ltd. (Beijing, China). All the chemical reagents were used without further purification.

\section{Synthesis of $\mathbf{L n}-\mathrm{SBD}$ and $\mathrm{Tb}_{1-x} \mathrm{Eu}_{x}$-SBD}

Lanthanide-doped SBD MOF was synthesized via a solvothermal reaction according to the previously reported literature with minor modifications [54]. Taking $5 \mathrm{~mol} \%$ $\mathrm{Tb}-\mathrm{SBD}$ as an example, $\mathrm{Sr}\left(\mathrm{NO}_{3}\right)_{2}(0.1058 \mathrm{~g}, 0.5 \mathrm{mmol})$, $\mathrm{Tb}\left(\mathrm{NO}_{3}\right)_{3} \cdot 5 \mathrm{H}_{2} \mathrm{O}(0.0109 \mathrm{~g}, 0.025 \mathrm{mmol}), \mathrm{BDC}(0.0831 \mathrm{~g}$, $0.5 \mathrm{mmol})$ and DMF $(16 \mathrm{~mL})$ were added to a $50-\mathrm{mL}$ beaker with vigorous stirring for $30 \mathrm{~min}$; the solution was transferred to a $50-\mathrm{mL}$ Teflon liner and sealed into a 50 -mL stainless steel autoclave, then heated at $140^{\circ} \mathrm{C}$ for 5 days. After being cooled to room temperature, colorless needle-shaped single crystals of Tb-SBD were washed carefully with DMF and dried under vacuum at $60^{\circ} \mathrm{C}$ for 10 h. Ln-SBD ( $\mathrm{Ln}=\mathrm{Dy}, \mathrm{Sm}, \mathrm{Eu})$ and $\mathrm{Tb}_{1-x} \mathrm{Eu}_{x}-\mathrm{SBD}(x=$ $0.2,0.4,0.6,0.8)$ were prepared in the similar procedure as $\mathrm{Tb}-\mathrm{SBD}$, except that the corresponding lanthanide nitrates $\mathrm{Ln}\left(\mathrm{NO}_{3}\right)_{3} \cdot x \mathrm{H}_{2} \mathrm{O}$ were added as luminescent centers. The nominal total concentration of lanthanide ions in $\mathrm{Ln}-\mathrm{SBD}$ and $\mathrm{Tb}_{1-x} \mathrm{Eu}_{x}-\mathrm{SBD}$ was constant, which was $10 \mathrm{~mol} \%$ of $\mathrm{Sr}^{2+}$ ions.

\section{Characterizations}

Powder X-ray diffraction (PXRD) measurements of the as-synthesized SBD and lanthanide-doped SBD were carried out in an X-ray diffractometer (Bruker, D8 ADVANCE) with $\mathrm{Cu} \mathrm{Ka}$ radiation $(\lambda=1.5406 \AA)$ at $40 \mathrm{kV}$ and $40 \mathrm{~mA}$. Rietveld refinement was performed by the total pattern analysis solution (TOPAS) Software 6. Both the simulated PXRD pattern of SBD and the starting model for refinement were derived from the CIF file (CCDC No. 817715) which could be downloaded from Cambridge Crystallographic Data Centre (CCDC) via www.ccdc.cam.ac.uk/structures [55]. The crystal structure was drawn by Diamond Software. Scanning electron microscopy (SEM) images were obtained by a tabletop scanning electron microscope (Hitachi, TM3000). Elemental mappings were obtained by using a field-emission scanning electron microscope (Hitachi, SU70) equipped with an energy dispersive X-ray (EDX) spectroscope system (Oxford Instruments, X-MaxN). Ultraviolet-visible spectroscopy (UV-Vis) and PL spectra were recorded at room temperature by spectrophotometer (Shimadzu, UV-3600 PLUS) and fluorescence spectrophotometer (Edinburgh Instruments, FLS980), respectively. Thermogravimetric analysis (TGA) was performed on a thermal analyzer (NETZSCH, TG209F1) with a heating rate of $10^{\circ} \mathrm{C} \mathrm{m^{-1 }}$. Fluorescence micrographs and confocal laser scanning images were obtained by using fluorescence microscope (Olympus, BX53M) and laser scanning confocal microscope (Leica, TCS SP8), respectively. ML spectra were recorded using a home-made measurement apparatus equipped with a fiber spectrometer (Ocean Optics, QE pro), optical fiber ( $\Phi=1000 \mu \mathrm{m}$ in diameter) and glass sleeve, wherein the optical fiber was inserted into the glass sleeve. Cathodoluminescence (CL) spectra were obtained by a scanning electron microscope (Zeiss, Sigma/VP) equipped with four micromanipulators (Kleindiek, Nanotechnik) and an imaging spectrometer (HORIBA, iHR320).

\section{Computational details}

All the calculations were performed using the Vienna $a b$ initio simulation package (VASP) [56]. Details of the crystal structure can be found in a previous report [55]. Projector augmented wave (PAW) and generalized gradient approximation (GGA) in the Perdew-Burke-Ernzerhof (PBE) form were adopted [57,58]. To ensure the precision of calculations, the electron wave functions were expanded in a plane wave basis with a kinetic energy cutoff of $600 \mathrm{eV}$. All the electrons were relaxed until the energy difference was less than $10^{-5} \mathrm{eV}$. The convergence threshold for structural optimization was set at $0.05 \mathrm{eV} \AA^{-1}$ in force. A $3 \times 3 \times 3$ Monkhorst-Pack grid was used for the sampling Brillouin zone [59]. VASPKIT was used for postprocessing [60].

\section{RESULTS AND DISCUSSION}

\section{Morphology and structures of SBD and Tb-SBD}

According to the crystal data, the SBD MOF belongs to the trigonal space group $P 3_{1}$ [55]. The framework has a distorted bi-capped coordination octahedron formed by the coordination of one $\mathrm{Sr}^{2+}$ ion with eight oxygen atoms, and two distinct triangular channels where the larger channels are filled with DMF molecules bound directly to the $\mathrm{Sr}^{2+}$ ions while the smaller ones are empty (Fig. 1a). Colorless needle-shaped single crystals of SBD (Fig. 1b) 

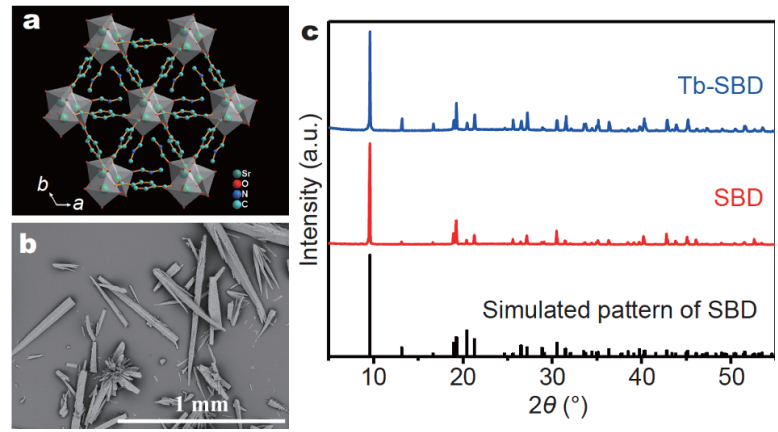

Figure 1 Crystal structure (a) and SEM image (b) of SBD. (c) PXRD patterns of the as-synthesized SBD and Tb-SBD.

were obtained, similar to those reported in the literature [55]. As shown in Fig. 1c, the diffraction peaks of the assynthesized SBD and Tb-SBD are narrow and strong, implying their high crystallinity. Meanwhile, all the diffraction peaks match well with the simulated pattern of SBD (CCDC No. 817715), which indicates not only the successful preparation of SBD crystals, but also there is no impurity phase formed after doping with $\mathrm{Tb}^{3+}$ ions. In other words, the $\mathrm{Tb}^{3+}$ ions were effectively incorporated into the SBD host.

To investigate the occupancy of $\mathrm{Tb}^{3+}$ ions in $\mathrm{Tb}-\mathrm{SBD}$, a series of tests including EDX mapping, fluorescence imaging, rietveld refinement and confocal laser scanning were carried out. The mapping data not only confirm the presence of $\mathrm{Tb}^{3+}$, but also further illustrate the uniform distribution of $\mathrm{Tb}^{3+}$ according to the almost overlapping elemental distribution of $\mathrm{Sr}, \mathrm{C}, \mathrm{O}$ and $\mathrm{Tb}$ (Fig. 2a). Fluorescence micrograph and confocal images further demonstrate the uniform distribution of $\mathrm{Tb}^{3+}$ ions in the host matrix (Fig. 2b and Fig. S1). Considering that $\mathrm{Tb}^{3+}$ dopants might be incorporated into the SBD lattice in two forms, either replacing the $\mathrm{Sr}^{2+}$ sites or entering the MOF channels, the Rietveld refinement of Tb-SBD in Fig. $2 \mathrm{c}$ was implemented by using TOPAS Software 6 , in which SBD (CCDC No. 817715) was taken as a starting model. As we can see, the experimental pattern is highly consistent with the calculated pattern, and the final refinement results are converged to $R_{\mathrm{wp}}$ (weighted profile residual $)=3.83 \%, R_{\mathrm{p}}$ (profile residual) $=4.01 \%$, GOF (goodness of fit) $=1.05$, indicating the high reliability of the Rietveld refinement. The atomic position data show that $\mathrm{Tb}^{3+}$ is incorporated into the SBD lattice by entering the $\mathrm{Sr}^{2+}$ site and the doping concentration is ca. $0.237 \mathrm{~mol} \%$ (Fig. $2 \mathrm{~d}$ and Table S1). In addition, compared with pure SBD, Tb-SBD exhibits slightly smaller cell parameters and cell volume (inserted table in Fig. 2c), which further confirms the successful substitution of $\mathrm{Sr}^{2+}$ (coordination number $\mathrm{CN}=8,1.26 \AA$,) by smaller $\mathrm{Tb}^{3+}$
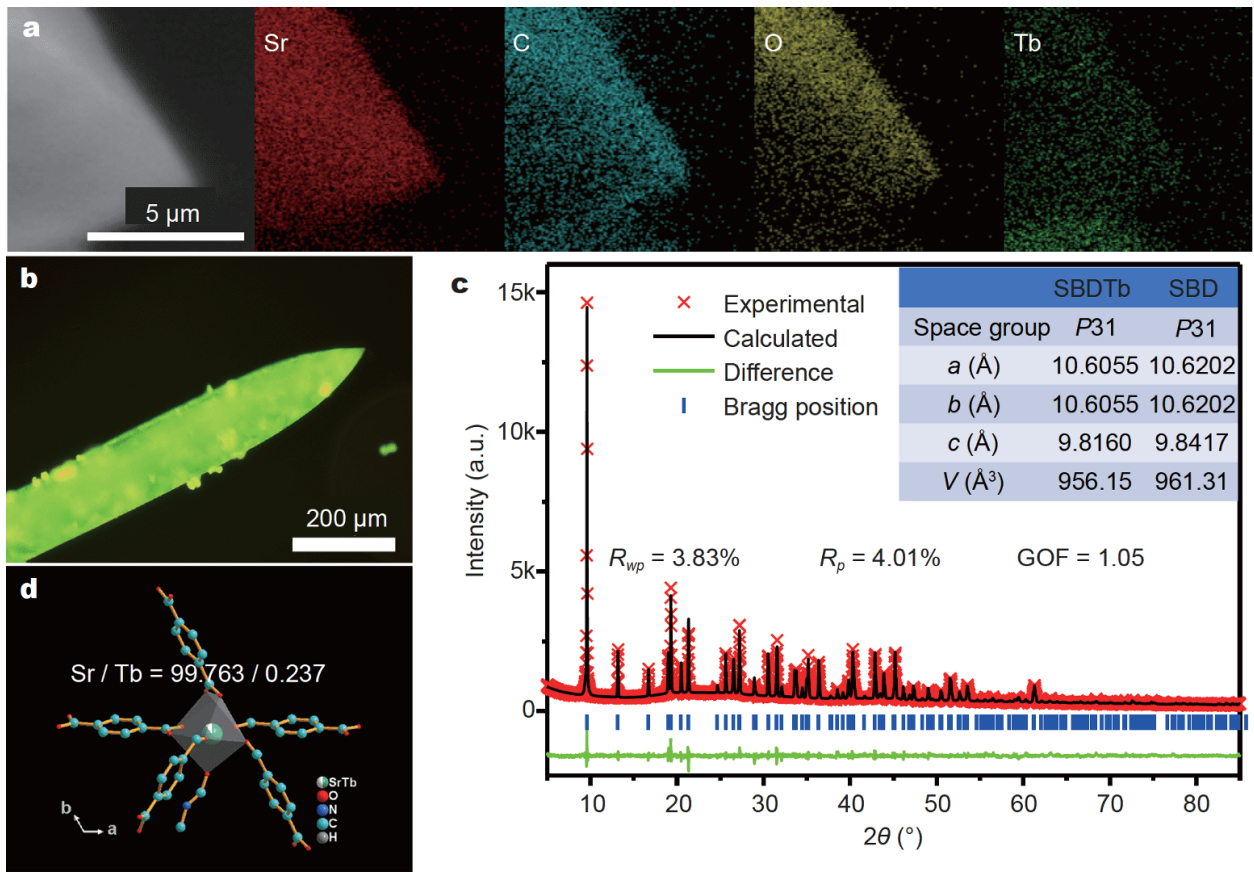

Figure 2 (a) EDX mapping, (b) fluorescence micrograph and (c) rietveld refinement of Tb-SBD. The inserted table in (c) shows the comparison of cell parameters before and after $\mathrm{Tb}$ doping. (d) Distorted bi-capped coordination octahedron of the $\mathrm{Sr}^{2+}$ and $^{\mathrm{T} b^{3+}}$ ions in $\mathrm{Tb}-\mathrm{SBD}$. 
ions $(\mathrm{CN}=8,1.04 \AA)$. Therefore, the $\mathrm{Tb}^{3+}$ ions are successfully introduced into the host lattice rather than being encapsulated into the pores of MOFs.

Besides, TGA plots of SBD and Tb-SBD in the temperature range of $50-750^{\circ} \mathrm{C}$ under air flow are shown in Fig. S2. SBD shows a weight loss of $\sim 23 \%$ at $350^{\circ} \mathrm{C}$, corresponding to the removal of coordinated DMF in the MOF pores, and the framework is not collapsed until $500^{\circ} \mathrm{C}$. These results are well consistent with the reported ones [55]. After doping with $\mathrm{Tb}^{3+}$, the collapse temperature of Tb-SBD is up to $440^{\circ} \mathrm{C}$.

\section{PL of SBD and Tb-SBD}

The band structure of pure SBD calculated by using VASP is presented in Fig. 3a. The results show that the valence band maximum and the conduction band minimum are located at different points, indicating that SBD has an indirect band gap. The band gap energy $\left(E_{\mathrm{g}}\right)$ was calculated to be approximately $3.28 \mathrm{eV}$. As we know, the analysis of density of states (DOS) is considered as an effective way to assign the band structure and analyze the bonding interactions in a crystal [61]. Herein, both valence bands are close to the Fermi level and the conduction bands below $3.5 \mathrm{eV}$ are dominated by states of $\mathrm{C}$ and $\mathrm{O}$. Therefore, the band gap of SBD is mainly determined by $\mathrm{C}$ and $\mathrm{O}$ atoms. Because these two elements are basically from the carboxyl group of the BDC ligand, the band gap of SBD is closely related to the BDC ligand.

UV-Vis diffuse reflectance spectrum of SBD is displayed in Fig. 3b. The decreasing reflectance of SBD from 250 to $350 \mathrm{~nm}$ is ascribed to the host absorption. On the basis of the Kubelka-Munk method and Tauc plot, the $E_{\mathrm{g}}$ of SBD can be estimated to be $3.75 \mathrm{eV}$ from the diffuse reflectance spectra, by plotting $[F(R) h v]^{1 / 2}$ (where $F(R)$ is KubelkaMunk function and $h v$ is photon energy) as a function of the photon energy (in $\mathrm{eV}$ ) and then extrapolating the linear portion to the photon energy axis (inset of Fig. 3b) $[62,63]$. Although this estimated $E_{\mathrm{g}}$ is slightly higher than the cal-
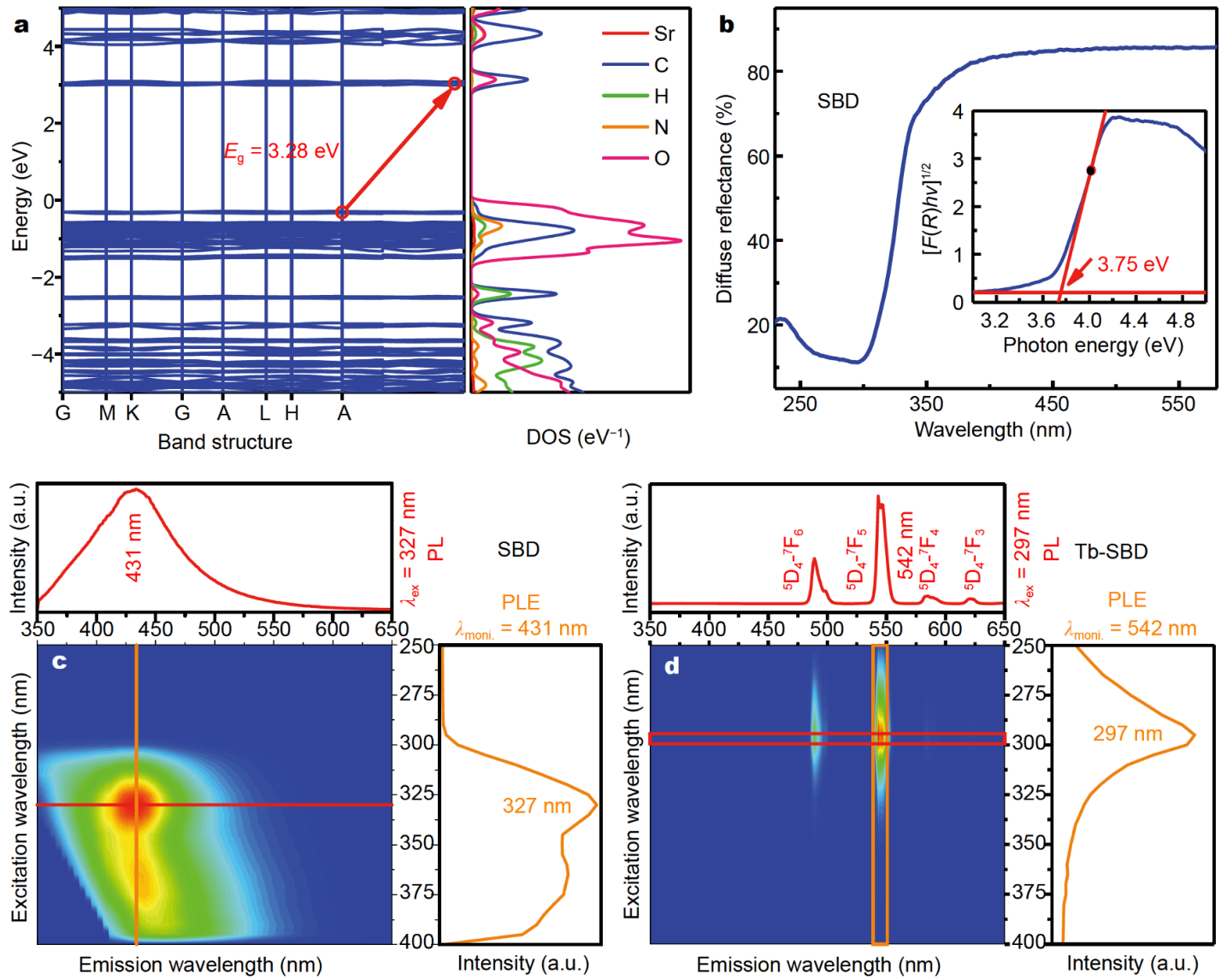

Figure 3 (a) Calculated band structure of SBD and the corresponding projected DOS on elements. (b) Diffuse reflectance spectra of SBD; the inset shows the corresponding Tauc plot. Excitation vs. emission wavelength contour plot for PL intensity in (c) SBD and (d) Tb-SBD, where PLE means PL excitation. 
culated $E_{\mathrm{g}}(3.28 \mathrm{eV})$, it is almost consistent with the energy of the excitation peak of the SBD host at $330 \mathrm{~nm}(3.76 \mathrm{eV})$.

Fig. $3 c$, d show the excitation versus emission wavelength contour plots for the PL intensity of SBD and TbSBD at room temperature, respectively. As shown in Fig. 3c, SBD exhibits a broad emission band between 350 and $550 \mathrm{~nm}$ under excitation in the spectral range of $300-400 \mathrm{~nm}$, which can be ascribed to the host luminescence caused by the $\pi-\pi$ electronic transition of the BDC ligand (Fig. S3). Among them, under the optimal excitation of $327 \mathrm{~nm}$, a strong emission at $431 \mathrm{~nm}$ can be observed. As we can see in Fig. 3d, a series of characteristic emission bands at 488, 542, 584 and $621 \mathrm{~nm}$ can be observed in the as-synthesized Tb-SBD, corresponding to the typical $4 \mathrm{f}-4 \mathrm{f}$ transitions of $\mathrm{Tb}^{3+}$ from ${ }^{5} \mathrm{D}_{4}$ to ${ }^{7} \mathrm{~F}_{J}(J=$ 6-3). Especially, when excited at $297 \mathrm{~nm}$, it shows the brightest green emission with a maximum of $542 \mathrm{~nm}$.

\section{ML of Tb-SBD}

To acquire ML spectrum more effectively, a measurement apparatus was built (Fig. S4) and the acquired ML spectra are displayed in Fig. 4a. Upon grinding, the as-prepared
Tb-SBD exhibits obvious green ML emission. Although the poorer signal to noise ratio in the ML spectrum indicates ML has a lower intensity than PL, both spectra are quite identical in shape and position. It implies that both ML and PL stem from the same excited state of $\mathrm{Tb}^{3+}$ ions [49]. A small deviation in emission peak is caused by using different spectrometers. In contrast, the pure SBD shows no $\mathrm{ML}$ even in the range of its strongest $\mathrm{PL}$ emission band $(431 \mathrm{~nm})$. The successful acquisition of ML phenomenon in Tb-SBD, to some extent, means that the strategy to discover novel ML-MOF materials by combination of a non-centrosymmetric MOF with lanthanide ions works well, which allows to realize the multifunctionalization of ML materials and to broaden the application of MOF materials.

To explore the ML mechanism of $\mathrm{Tb}-\mathrm{SBD}$, the following investigations were carried out. As we can see, the needle-like Tb-SBD samples were severely damaged after vigorous grinding (Fig. S5a), and no ML emission can be seen when grinded again. It means that $\mathrm{Tb}-\mathrm{SBD}$ is essentially an FML material that exhibits ML at the expense of crystal fracture, resembling most reported metal-
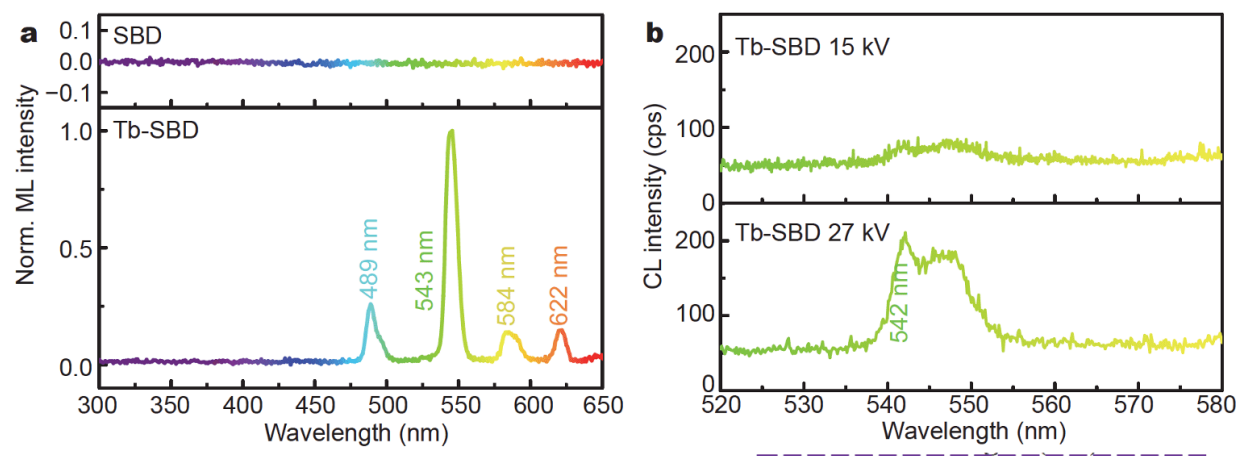

c
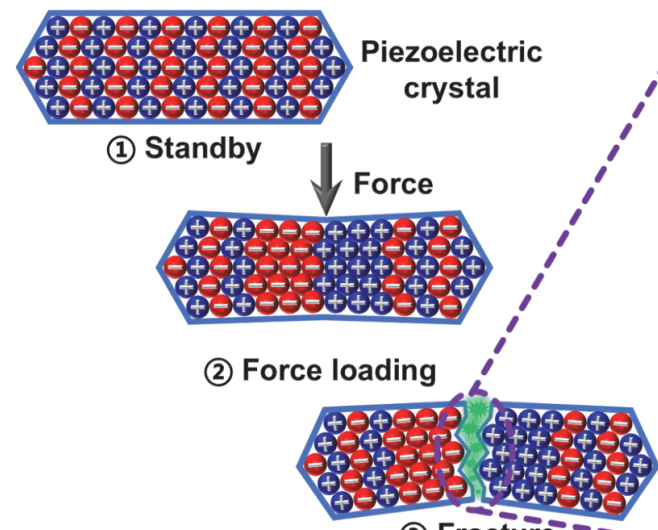

(3) Fracture

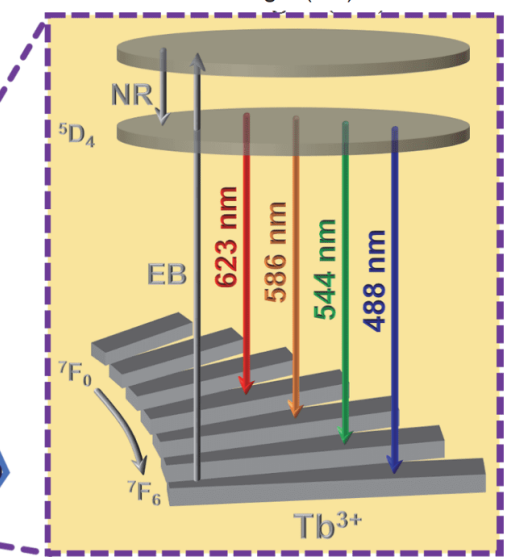

Figure 4 (a) ML spectra of SBD and Tb-SBD upon grinding. (b) CL spectra of Tb-SBD under different accelerating voltages. (c) Schematic of ML mechanism in Tb-SBD. 
organic complexes and ML-MOFs [28-32,45-51]. Besides, there is no change in the phase after grinding (Fig. $\mathrm{S} 5 \mathrm{~b}$ ), which rules out that the ML of Tb-SBD is caused by the phase transition-induced chemiluminescence.

Although there are no systematic studies on the FML mechanism of ML-MOFs yet, the relatively mature mechanisms in other FML materials can be used to understand the FML behavior of Tb-SBD [42-51,64-67]. Chandra et al. $[42,43]$ have proposed 21 possible ML models, of which the electron bombardment (EB) model is one of the most recognized one. The excitation energy required to excite the PL of dinitrogen is usually larger than that of most luminescence centers (such as lanthanide ions), and the energy of the electron emission produced during fracture has also been proved to be sufficient to excite solid-state luminescence [43]. Hence, the luminescence centers in ML materials could also be directly excited by the EB process, in a similar way that the dinitrogen is excited $\left({ }^{3} \pi_{\mathrm{u}} \rightarrow{ }^{3} \pi_{\mathrm{g}}, 280-440 \mathrm{~nm}\right)$ [43], which can be verified by the CL performance of ML materials [43].

Since the excitation spectra of SBD and Tb-SBD overlap with the gas discharge spectrum in a large range, as long as the gas discharge occurs, both SBD and Tb-SBD will exhibit corresponding solid-state luminescence via the effective energy transfer (ET) process. In fact, pure SBD shows neither gas discharge ML nor solid-state ML corresponding to BDC ligands and Tb-SBD shows only solid-state $\mathrm{ML}$ corresponding to $\mathrm{Tb}^{3+}$ ions. Therefore, the absence of dinitrogen lines in these two ML spectra should not be due to the reabsorption, but due to the insufficient electric field for gas discharge. According to the point of view mentioned above, the green $\mathrm{ML}$ of $\mathrm{Tb}$ SBD is most likely caused by the direct bombardment of $\mathrm{Tb}^{3+}$ ions by high-energy electrons. Fig. $4 \mathrm{~b}$ shows that the Tb-SBD sample has a clear CL emission (542 nm) similar to $\mathrm{PL}$ at high accelerating voltage, corresponding to the transition of $\mathrm{Tb}^{3+}$ ions from ${ }^{5} \mathrm{D}_{4}$ to ${ }^{7} \mathrm{~F}_{5}$, but not significant at low accelerating voltage. This is because the higher accelerating voltage $(27 \mathrm{kV})$ could provide EB energy high enough to directly excite $\mathrm{Tb}^{3+}$ ions to form effective $\mathrm{CL}$ emission, while the lower accelerating voltage $(15 \mathrm{kV})$ cannot. The results well support the point of view that the luminescence centers in ML materials could be directly excited by the EB process, and further confirm that the $\mathrm{EB}$ mechanism can be used to explain the ML of Tb-SBD.

Consequently, it is proposed that the FML process in Tb-SBD happens in the following steps (Fig. 5c). Firstly, Tb-SBD MOF, a fragile piezoelectric crystal with acentric space group $P 3_{1}$, shows a uniform charge distribution when there is no force loading (standby mode). Secondly, when a minute force is loaded, the charge separation occurs due to the piezoelectrification resulting from the acentric structure of Tb-SBD, and thus a large amount of separated charges appear (force loading mode). It is this critical process that lays a decisive foundation for the intense ML of Tb-SBD. In other words, it is the piezoelectric effect, derived from the non-centrosymmetric structure, that effectively promotes the charge separation before the fracture of the Tb-SBD crystals, thereby resulting in the intense ML visible to the naked eyes. Thirdly, with increasing stress, the needle-like crystals crack and fracture rapidly, generating countless new charged surfaces, following the Langevin model [43] (fracture mode). When the electric field produced between the charged surfaces reaches the order of $10^{7}$ $10^{8} \mathrm{~V} \mathrm{~m}^{-1}$, EB occurs between these opposite surfaces $[42,43]$. The energy released by this process directly excites the ground state electrons of $\mathrm{Tb}^{3+}$ to the excited state, thereby producing luminescence by radiative transition, which is similar to CL. It should be noted that since there is no effective ET from ligand to metal in Tb$\mathrm{SBD}$, the contribution of the ET process to ML can be neglected, so the influence of the BDC ligand on ML has not been mentioned in the above discussion. For comparison, two Tb-doped centrosymmetric MOFs different from Tb-SBD were prepared (Fig. S6a) $[54,68]$. The results shown in Fig. S6b reveal that there are no visible ML emissions in them, which further confirms the close relationship between the intense ML of Tb-SBD and its non-centrosymmetric structure.

Multicolor mechanoluminescence of $\operatorname{Ln}-\mathrm{SBD}(\mathrm{Ln}=\mathrm{Tb}, \mathrm{Dy}$, $\mathrm{Sm}, \mathrm{Eu})$ and $\mathrm{Tb}_{1-x} \mathrm{Eu}_{x}-\mathrm{SBD}(x=0.2,0.4,0.6,0.8)$

Inspired by the successful synthesis of Tb-SBD, a series of lanthanide-doped MOFs Ln-SBD ( $\mathrm{Ln}=\mathrm{Tb}, \mathrm{Dy}, \mathrm{Sm}, \mathrm{Eu})$ and $\mathrm{Tb}_{1-x} \mathrm{Eu}_{x}$-SBD $(x=0.2,0.4,0.6,0.8)$ were successfully synthesized as well (Fig. S7), and their ML spectra were measured (Fig. 5). As shown in Fig. 5a, b, the as-synthesized $\operatorname{Ln}-\mathrm{SBD}(\mathrm{Ln}=\mathrm{Tb}, \mathrm{Dy}, \mathrm{Sm}, \mathrm{Eu})$ display $\mathrm{ML}$ emissions of green $\left(\mathrm{Tb}^{3+}\right)$ yellow $\left(\mathrm{Dy}^{3+}\right)$, orange $\left(\mathrm{Sm}^{3+}\right)$ and red $\left(\mathrm{Eu}^{3+}\right)$. Similarly, with the increase of $\mathrm{Eu}^{3+}$ contents, the as-synthesized $\mathrm{Tb}_{1-x} \mathrm{Eu}_{x}$-SBDs $(x=0,0.2,0.4$, $0.6,0.8,1)$ also display multicolor ML emissions from green to red (Fig. $5 \mathrm{c}-\mathrm{e}$ ). In a word, the as-synthesized lanthanide-doped MOFs exhibit multicolor ML emissions from green to yellow to red by changing the co-doping ratios and species of lanthanide ions. To the best of our knowledge, it is the first time to achieve multicolor $\mathrm{ML}$ emissions in MOF materials. 

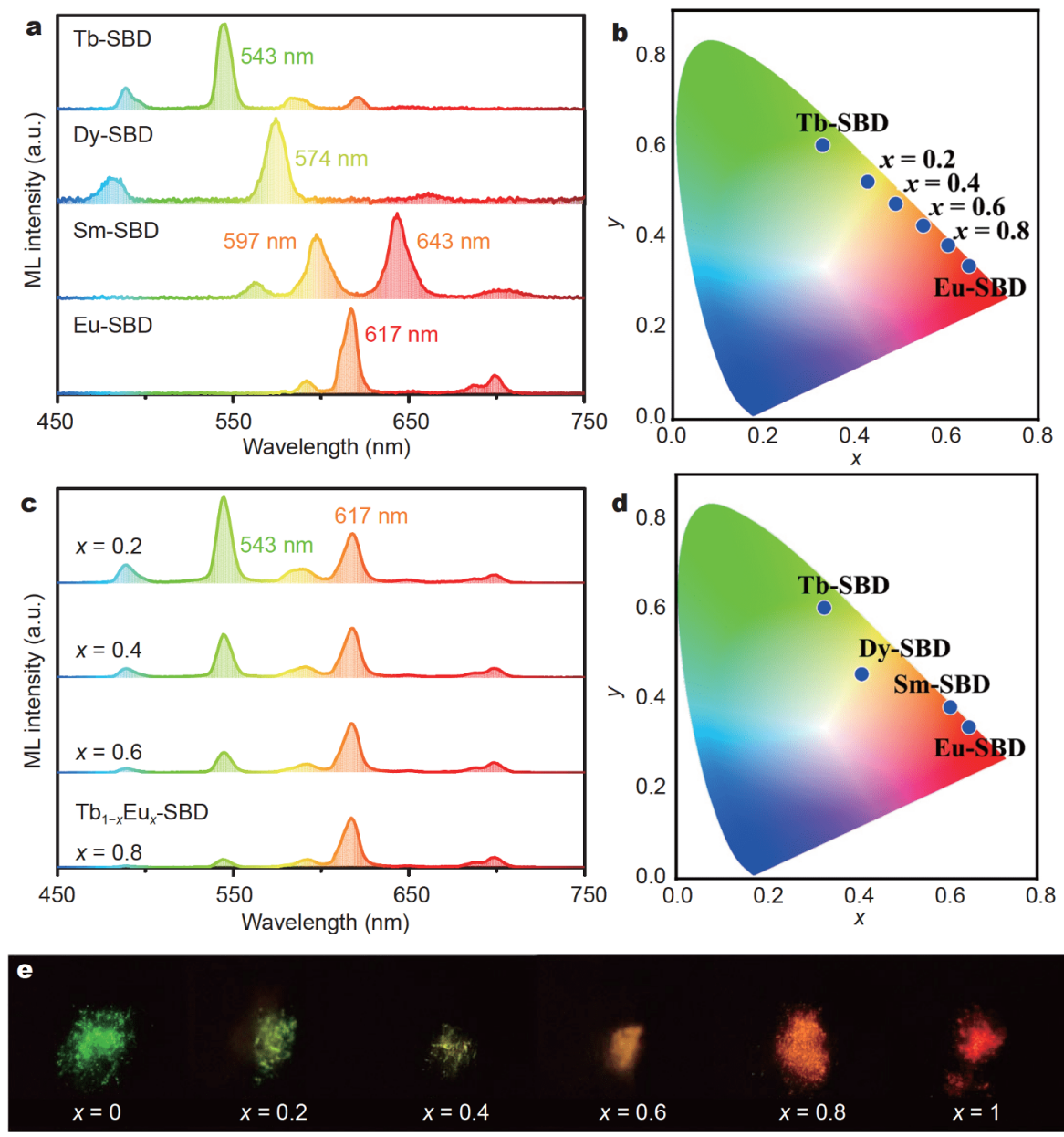

Figure 5 (a) Multicolor ML spectra and (b) Commission Internationale de l'Eclairage (CIE) chromaticity coordinates of Ln-SBD (Ln = Tb, Dy, Sm, $\mathrm{Eu})$. (c) Multicolor ML spectra, (d) CIE chromaticity coordinates and (e) ML images of $\mathrm{Tb}_{{ }_{1-x}} \mathrm{Eu}_{x}$-SBD $(x=0.2,0.4,0.6,0.8)$.

\section{Temperature sensing based on ML}

To realize the combination of stress detection and temperature sensing, $\mathrm{Tb}_{0.8} \mathrm{Eu}_{0.2}$-SBD was used as an example to explore its temperature sensing performance based on ML. As shown in Fig. 6a, ML intensity ratios of $I_{618} / I_{544}$ in $\mathrm{Tb}_{0.8} \mathrm{Eu}_{0.2}$-SBD change with temperature, suggesting that temperature has different impacts on the luminescence center $\mathrm{Tb}^{3+}\left({ }^{5} \mathrm{D}_{4} \rightarrow{ }^{7} \mathrm{~F}_{5}, 544 \mathrm{~nm}\right)$ and $\mathrm{Eu}^{3+}\left({ }^{5} \mathrm{D}_{0} \rightarrow{ }^{7} \mathrm{~F}_{2}\right.$, $618 \mathrm{~nm}$ ). Fig. $6 \mathrm{~b}$ clearly shows that the ML intensity ratio gradually increases with increasing temperature. More importantly, the relationship between ML intensity ratio $I_{618} / I_{544}$ and temperature could be fitted as a function:

$\frac{I_{618}}{I_{544}}=6067 \mathrm{e}^{-5717 / T}+0.4484$,

with a correlation coefficient of $R^{2}=0.991$, where $I_{618}$ and $I_{544}$ are the $\mathrm{ML}$ intensity of $\mathrm{Eu}^{3+}$ and $\mathrm{Tb}^{3+}$, respectively, and $T$ refers to the absolute temperature. To judge the temperature sensing performance of different thermometers, the relative sensitivity $(\mathrm{Sr})$ could be determined as $[52,53]$ :

$\mathrm{Sr}=\frac{\mathrm{d}\left(I_{618} / I_{544}\right) / \mathrm{d} T}{I_{618} / I_{544}}$.

According to the Equations (1) and (2), the Sr of $\mathrm{Tb}_{0.8} \mathrm{Eu}_{0.2}$ SBD in the range of $298-563 \mathrm{~K}$ increases monotonically, and the maximum $\mathrm{Sr}$ is determined to be $0.621 \% \mathrm{~K}^{-1}$ at $563 \mathrm{~K}$ (Fig. 6c). Although the sensitivity is undesirable compared with the existing luminescent thermometers [15-18,52,53], as far as we know, it is the first temperature sensing research based on ML, which has important guiding significance for the subsequent similar research.

\section{CONCLUSIONS}

In summary, a strategy for developing ML-MOFs by 

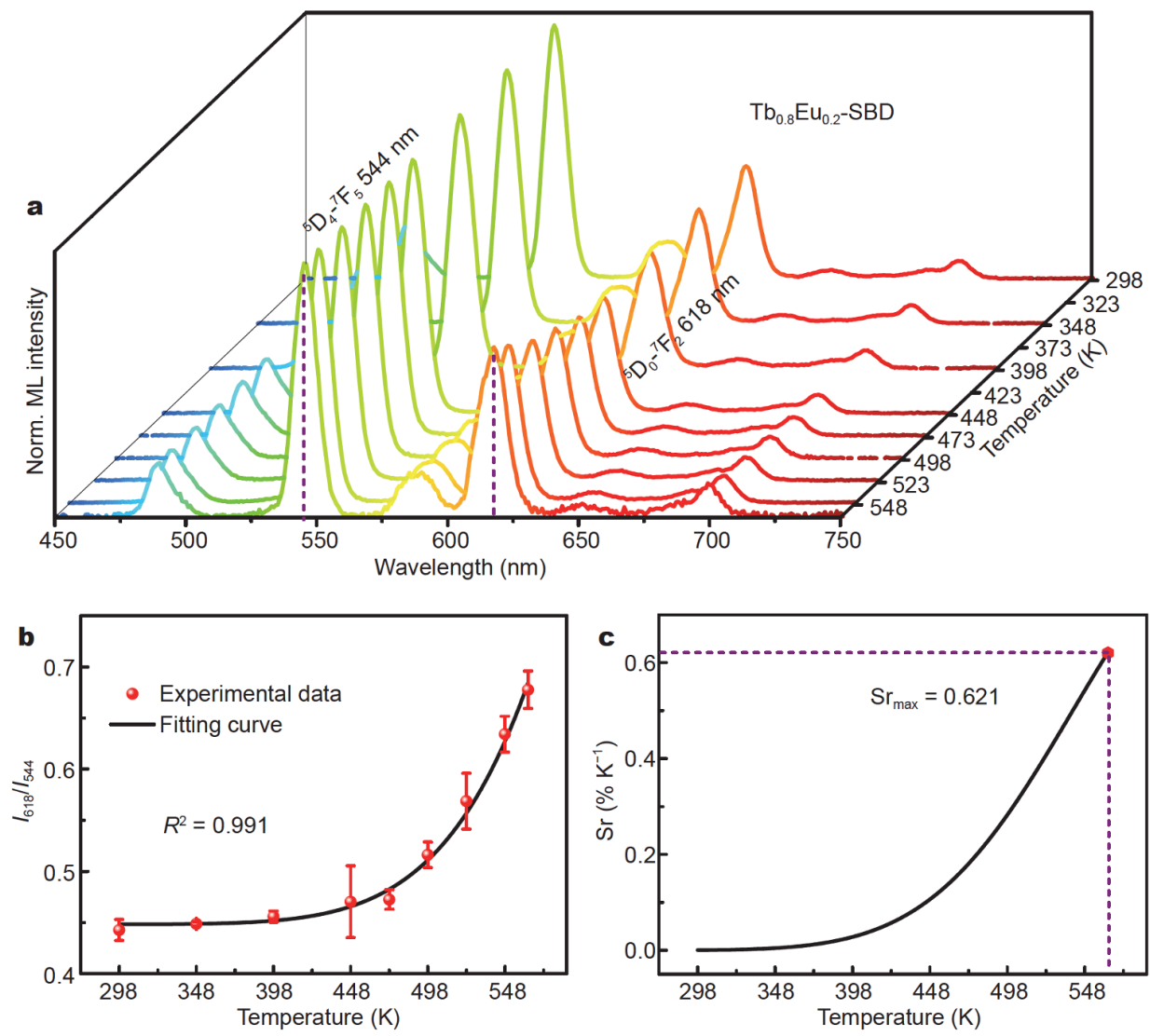

Figure 6 (a) ML spectra of $\mathrm{Tb}_{0.8} \mathrm{Eu}_{0.2} \mathrm{SBD}$ at different temperatures which are normalized at $544 \mathrm{~nm}$. (b) $\mathrm{ML}$ intensity ratio $\left(I_{618} / I_{544}\right)$ of $\mathrm{Tb}_{0.8} \mathrm{Eu}_{0.2}$ SBD as a function of temperature (red dots); white line is the fitting curve and all error bars are based on means \pm standard deviation $(n=3)$. (c) Relative sensitivity of temperature sensing based on the ML intensity ratio of Eu/Tb in $\mathrm{Tb}_{0.8} \mathrm{Eu}_{0.2}-\mathrm{SBD}$.

doping lanthanide ions into the non-centrosymmetric SBD MOF was proposed, and a series of lanthanidedoped MOFs Ln-SBD $(\mathrm{Ln}=\mathrm{Tb}, \mathrm{Dy}, \mathrm{Sm}, \mathrm{Eu})$ and $\mathrm{Tb}_{1-x} \mathrm{Eu}_{x}$-SBD $(x=0.2,0.4,0.6,0.8)$ with multicolor $\mathrm{ML}$ were successfully synthesized by this strategy. SBD is a piezoelectric MOF with an indirect band gap and the $E_{\mathrm{g}}$ (calculated: $3.28 \mathrm{eV}$; UV-Vis: $3.75 \mathrm{eV}$ ) is closely related to the $\mathrm{BDC}$ ligand. $\mathrm{Tb}^{3+}$ ions doping did not destroy the excellent thermal stability of the SBD matrix, and also yields bright green PL of $\mathrm{Tb}^{3+}$. Very interestingly, strong green ML emission similar to PL in Tb-SBD was observed. The intense ML emission could be caused by the direct excitation of lanthanide ions from the EB process, while the EB process can be attributed to the piezoelectric effect of the non-centrosymmetric SBD host. In addition, yellow, orange and red MLs were also obtained by changing the co-doping ratios and species of lanthanide ions. It is the first time to achieve multicolor ML from green to yellow to red in a MOF host. Herein, the successful acquisition of ML-MOFs indicates that the strat- egy of combining a non-centrosymmetric MOF host with lanthanide ions is successful and feasible, which is of great significance not only for the development of more lanthanide-doped ML-MOFs, but also for broadening the application of MOF materials and realizing multifunction of ML materials. Finally, a pioneering temperature sensing research based on ML was carried out with the obtained ML-MOFs. Although the temperature sensing sensitivity was undesirable $\left(0.621 \% \mathrm{~K}^{-1}\right)$, it is the first combination of stress detection and temperature sensing, which is of great significance for realizing dual-functional detection of stress and temperature without excitation light source and the subsequent similar research.

Received 18 July 2020; accepted 24 August 2020; published online 15 December 2020

1 Li H, Eddaoudi M, O'Keeffe M, et al. Design and synthesis of an exceptionally stable and highly porous metal-organic framework. Nature, 1999, 402: 276-279

2 Eddaoudi M, Kim J, Rosi N, et al. Systematic design of pore size 
and functionality in isoreticular MOFs and their application in methane storage. Science, 2002, 295: 469-472

3 Yaghi OM, O'Keeffe M, Ockwig NW, et al. Reticular synthesis and the design of new materials. Nature, 2003, 423: 705-714

4 Furukawa H, Cordova KE, O'Keeffe M, et al. The chemistry and applications of metal-organic frameworks. Science, 2013, 341: 1230444

5 Rowsell JLC, Spencer EC, Eckert J, et al. Gas adsorption sites in a large-pore metal-organic framework. Science, 2005, 309: 1350-1354

6 Kim H, Yang S, Rao SR, et al. Water harvesting from air with metal-organic frameworks powered by natural sunlight. Science, 2017, 356: 430-434

7 Rosi NL, Eckert J, Eddaoudi M, et al. Hydrogen storage in microporous metal-organic frameworks. Science, 2003, 300: 1127-1129

8 Liang Z, Qu C, Guo W, et al. Pristine metal-organic frameworks and their composites for energy storage and conversion. Adv Mater, 2018, 30: 1702891

9 Lee JY, Farha OK, Roberts J, et al. Metal-organic framework materials as catalysts. Chem Soc Rev, 2009, 38: 1450-1459

10 Wu MX, Yang YW. Metal-organic framework (MOF)-based drug/ cargo delivery and cancer therapy. Adv Mater, 2017, 29: 1606134

11 Kreno LE, Leong K, Farha OK, et al. Metal-organic framework materials as chemical sensors. Chem Rev, 2012, 112: 1105-1125

12 Zhang T, Lin W. Metal-organic frameworks for artificial photosynthesis and photocatalysis. Chem Soc Rev, 2014, 43: 5982-5993

13 Fang X, Shang Q, Wang Y, et al. Single Pt atoms confined into a metal-organic framework for efficient photocatalysis. Adv Mater, 2018, 30: 1705112

14 Yan B. Lanthanide-functionalized metal-organic framework hybrid systems to create multiple luminescent centers for chemical sensing. Acc Chem Res, 2017, 50: 2789-2798

15 Rao X, Song T, Gao J, et al. A highly sensitive mixed lanthanide metal-organic framework self-calibrated luminescent thermometer. J Am Chem Soc, 2013, 135: 15559-15564

16 Cui Y, Song R, Yu J, et al. Dual-emitting MOFつdye composite for ratiometric temperature sensing. Adv Mater, 2015, 27: 1420-1425

17 Cui Y, Xu H, Yue Y, et al. A luminescent mixed-lanthanide metalorganic framework thermometer. J Am Chem Soc, 2012, 134: 3979-3982

18 Cui Y, Zhu F, Chen B, et al. Metal-organic frameworks for luminescence thermometry. Chem Commun, 2015, 51: 7420-7431

19 Liu J, Zhuang Y, Wang L, et al. Achieving multicolor long-lived luminescence in dye-encapsulated metal-organic frameworks and its application to anticounterfeiting stamps. ACS Appl Mater Interfaces, 2018, 10: 1802-1809

20 Li Z, Wang G, Ye Y, et al. Loading photochromic molecules into a luminescent metal-organic framework for information anticounterfeiting. Angew Chem Int Ed, 2019, 58: 18025-18031

$21 \mathrm{Yu}$ J, Cui $\mathrm{Y}, \mathrm{Xu} \mathrm{H}$, et al. Confinement of pyridinium hemicyanine dye within an anionic metal-organic framework for two-photonpumped lasing. Nat Commun, 2013, 4: 2719

$22 \mathrm{He} \mathrm{H}, \mathrm{Ma}$ E, Cui Y, et al. Polarized three-photon-pumped laser in a single MOF microcrystal. Nat Commun, 2016, 7: 11087

23 Wei Y, Dong H, Wei C, et al. Wavelength-tunable microlasers based on the encapsulation of organic dye in metal-organic frameworks. Adv Mater, 2016, 28: 7424-7429

24 Medishetty R, Nalla V, Nemec L, et al. A new class of lasing materials: Intrinsic stimulated emission from nonlinear optically active metal-organic frameworks. Adv Mater, 2017, 29: 1605637 fluorescent dyes into a metal-organic framework for tunable white light emission. Adv Mater, 2017, 29: 1700778

26 Chen W, Zhuang Y, Wang L, et al. Color-tunable and high-efficiency dye-encapsulated metal-organic framework composites used for smart white-light-emitting diodes. ACS Appl Mater Interfaces, 2018, 10: 18910-18917

27 Wang Z, Zhu CY, Mo JT, et al. White-light emission from dualway photon energy conversion in a dye-encapsulated metalorganic framework. Angew Chem Int Ed, 2019, 58: 9752-9757

28 Yuan L, Yin M, Yuan E, et al. Syntheses, structures and luminescence of europium $\alpha$-thiophene carboxylates coordination polymer and supramolecular compound. Inorg Chim Acta, 2004, 357: 89-94

29 Eliseeva SV, Pleshkov DN, Lyssenko KA, et al. Highly luminescent and triboluminescent coordination polymers assembled from lanthanide $\beta$-diketonates and aromatic bidentate $\mathrm{O}$-donor ligands. Inorg Chem, 2010, 49: 9300-9311

30 Hasegawa Y, Hieda R, Miyata K, et al. Brilliant triboluminescence of a lanthanide coordination polymer with low-vibrational-frequency and non-centrosymmetric structural networks. Eur J Inorg Chem, 2011, 2011(32): 4978-4984

31 Hasegawa Y, Tateno S, Yamamoto M, et al. Effective photo- and triboluminescent europium(III) coordination polymers with rigid triangular spacer ligands. Chem Eur J, 2017, 23: 2666-2672

32 Hirai Y, Nakanishi T, Kitagawa Y, et al. Triboluminescence of lanthanide coordination polymers with face-to-face arranged substituents. Angew Chem Int Ed, 2017, 56: 7171-7175

33 Bacon F. The advancement of learning [1605]. In: Devey J (ed). Fourth Book, Chapter III. New York: P.F. Collier and Son, 1901

34 Herschel AS. Triboluminescence. Nature, 1899, 60: 29

35 Sage I, Bourhill G. Triboluminescent materials for structural damage monitoring. J Mater Chem, 2001, 11: 231-245

36 Wang $\mathrm{X}$, Zhang $\mathrm{H}, \mathrm{Yu}$ R, et al. Dynamic pressure mapping of personalized handwriting by a flexible sensor matrix based on the mechanoluminescence process. Adv Mater, 2015, 27: 2324-2331

37 Peng D, Chen B, Wang F. Recent advances in doped mechanoluminescent phosphors. ChemPlusChem, 2015, 80: 1209-1215

$38 \mathrm{Tu} \mathrm{D}, \mathrm{Xu} \mathrm{CN}$, Yoshida A, et al. $\mathrm{LiNbO}_{3}: \mathrm{Pr}^{3+}$ : A multipiezo material with simultaneous piezoelectricity and sensitive piezoluminescence. Adv Mater, 2017, 29: 1606914

39 Du Y, Jiang Y, Sun T, et al. Mechanically excited multicolor luminescence in lanthanide ions. Adv Mater, 2019, 31: 1807062

40 Liu L, Xu CN, Yoshida A, et al. Scalable elasticoluminescent strain sensor for precise dynamic stress imaging and onsite infrastructure diagnosis. Adv Mater Technol, 2019, 4: 1800336

41 Chen $\mathrm{C}$, Zhuang $\mathrm{Y}, \mathrm{Tu} \mathrm{D}$, et al. Creating visible-to-near-infrared mechanoluminescence in mixed-anion compounds $\mathrm{SrZn}_{2} \mathrm{~S}_{2} \mathrm{O}$ and SrZnSO. Nano Energy, 2020, 68: 104329

42 Chandra BP, Rathore AS. Classification of mechanoluminescence. Cryst Res Technol, 1995, 30: 885-896

43 Chandra BP, Chandra VK, Jha P. Models for intrinsic and extrinsic fracto-mechanoluminescence of solids. J Lumin, 2013, 135: 139153

44 Jha P, Chandra BP. Survey of the literature on mechanoluminescence from 1605 to 2013. Luminescence, 2014, 29: 977-993

45 Hurt CR, Mcavoy N, Bjorklund S, et al. High intensity triboluminescence in europium tetrakis (dibenzoylmethide)-triethylammonium. Nature, 1966, 212: 179-180

46 Liu M, Wu Q, Shi H, et al. Progress of research on organic/organometallic mechanoluminescent materials. Acta Chim Sin, 2018, 76: $246-258$ 
47 Bünzli JCG, Wong KL. Lanthanide mechanoluminescence. J Rare Earths, 2018, 36: 1-41

48 Sweeting LM, Rheingold AL. Crystal disorder and triboluminescence: Triethylammonium tetrakis(dibenzoylmethanato)europate. J Am Chem Soc, 1987, 109: 2652-2658

49 Rheingold AL, King W. Crystal structures of three brilliantly triboluminescent centrosymmetric lanthanide complexes: Piperidinium tetrakis(benzoylacetonato)europate, hexakis(antipyrine) terbium triiodide, and hexaaquadichloroterbium chloride. Inorg Chem, 1989, 28: 1715-1719

50 Takada N, Hieda S, Sugiyama J, et al. Mechanoluminescence from piezoelectric crystals of an europium complex. Synth Met, 2000, 111-112: 587-590

51 Hasegawa Y, Kitagawa Y, Nakanishi T. Effective photosensitized, electrosensitized, and mechanosensitized luminescence of lanthanide complexes. NPG Asia Mater, 2018, 10: 52-70

52 Brites CDS, Balabhadra S, Carlos LD. Lanthanide-based thermometers: At the cutting-edge of luminescence thermometry. Adv Opt Mater, 2019, 7: 1801239

53 Wang X, Wolfbeis OS, Meier RJ. Luminescent probes and sensors for temperature. Chem Soc Rev, 2013, 42: 7834

54 Williams CA, Blake AJ, Wilson C, et al. Novel metal-organic frameworks derived from Group II metal cations and aryldicarboxylate anionic ligands. Cryst Growth Des, 2008, 8: 911-922

55 Pan C, Nan J, Dong X, et al. A highly thermally stable ferroelectric metal-organic framework and its thin film with substrate surface nature dependent morphology. J Am Chem Soc, 2011, 133: 12330-12333 Kresse G, Furthmüller J. Efficient iterative schemes for $a b$ initio total-energy calculations using a plane-wave basis set. Phys Rev B, 1996, 54: 11169-11186

57 Blöchl PE. Projector augmented-wave method. Phys Rev B, 1994, 50: $17953-17979$

58 Perdew JP, Burke K, Ernzerhof M. Generalized gradient approximation made simple. Phys Rev Lett, 1996, 77: 3865-3868

59 Monkhorst HJ, Pack JD. Special points for brillouin-zone integrations. Phys Rev B, 1976, 13: 5188-5192

60 Wang V, Xu N, Liu JC, et al. VASPKIT: A pre- and post-processing program for VASP code. 2019, arXiv: 1908.08269

61 Guo Q, Wang Q, Jiang $\mathrm{L}$, et al. A novel apatite, $\mathrm{Lu}_{5}\left(\mathrm{SiO}_{4}\right)_{3} \mathrm{~N}$ :(Ce, $\mathrm{Tb})$, phosphor material: Synthesis, structure and applications for NUV-LEDs. Phys Chem Chem Phys, 2016, 18: 15545-15554

62 Dorenbos P. Systematic behaviour in trivalent lanthanide charge transfer energies. J Phys-Condens Matter, 2003, 15: 8417-8434

63 Dorenbos P. The $\mathrm{Eu}^{3+}$ charge transfer energy and the relation with the band gap of compounds. J Lumin, 2005, 111: 89-104

64 Lin SH, Wutz D, Ho ZZ, et al. Mechanisms of triboluminescence. Proc Natl Acad Sci USA, 1980, 77: 1245-1247

65 Chandra BP. Mechanoluminescence and piezoelectric behaviour of molecular crystals. Phys Stat Sol A, 1981, 64: 395-405

66 Eddingsaas NC, Suslick KS. Light from sonication of crystal slurries. Nature, 2006, 444: 163

67 Mukherjee S, Thilagar P. Renaissance of organic triboluminescent materials. Angew Chem Int Ed, 2019, 58: 7922-7932

68 Guo X, Zhu G, Li Z, et al. A lanthanide metal-organic framework with high thermal stability and available Lewis-acid metal sites. Chem Commun, 2006, 3172-3174

Acknowledgements This work was supported by the National Natural Science Foundation of China (51832005). The authors also thank Dr. Pengfei Liu and Weibin Ye for their help in DFT calculation and CL characterizations, respectively.

Author contributions Xie RJ, Zhuang Y and Chen W conceived and designed the project, wrote and revised the paper; Chen $\mathrm{W}$ was primarily responsible for the experiments; Lv Y, Chen C and Wang MS helped revise this article, perform ML measurements and CL characterizations, respectively. All authors contributed to the general discussion.

Conflict of interest The authors declare that they have no conflict of interest.

Supplementary information online version of the paper.

Supporting data are available in the

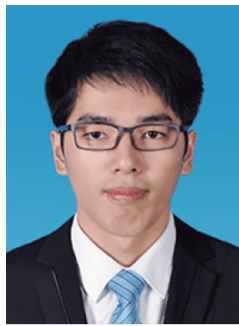

Wenwei Chen is currently a $\mathrm{PhD}$ student at the College of Materials, Xiamen University. $\mathrm{He}$ completed his master degree in 2016 from Xiamen University. His current research focuses on luminescent MOF materials.

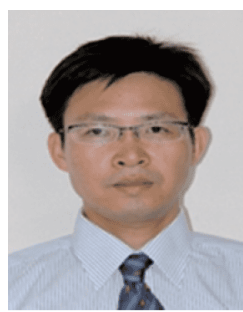

Rong-Jun Xie received his master degree in 1995 from Xi'an Jiaotong University, and received his $\mathrm{PhD}$ degree in 1998 from Shanghai Institute of Ceramics, Chinese Academy of Sciences. He is now a professor in the College of Materials, Xiamen University. His current research focuses on the application of rare earth doped luminescent materials and semiconductor lighting devices.

\section{多色应力发光的镧系掺杂金属有机框架}

陈文威 ${ }^{1,2}$, 庄逸熙 ${ }^{1,2^{*}}$, 陈昌健 ${ }^{1,2}$, 吕营 ${ }^{1,2}$, 王鸣生 ${ }^{1}$, 解荣军 ${ }^{1,2^{*}}$

摘要 金属有机框架 $(\mathrm{MOF})$ 和应力发光 $(\mathrm{ML})$ 材料被认为是两种有 前途的材料, 并在诸多领域得到了广泛应用. 若能将二者结合起来 获得ML-MOF材料, 则势必会拓展它们的应用范围. 但目前对MLMOF的研究并不多, 且其中的ML机制仍不明确. 在本研究中, 我们 提出了一种通过在非中心对称SBD MOF中掺杂镧系离子来开发 ML-MOF的策略, 成功合成了一系列具有多色应力发光的镧系掺 杂MOF Ln-SBD ( $\mathrm{Ln}=\mathrm{Tb}, \mathrm{Dy}, \mathrm{Sm}, \mathrm{Eu})$ 和 $\mathrm{Tb}_{1-x} \mathrm{Eu}_{x}-\mathrm{SBD}(x=0.2$, $0.4,0.6,0.8)$. 锞系离子均匀分布在 $\mathrm{SBD}$ 基质中, 并部分占据了 $\mathrm{Sr}$ 的 格位. 通过改变共掺比例和掺杂种类, ML-MOF表现出了从绿色到 黄色再到红色的多色应力发光. 相似的ML和PL光谱表明, ML发射 与镧系离子从激发态到基态的辐射跃迁有关. 辐射跃迁是由电子 轰击过程引起的, 而电子轰击过程源于非中心对称 $\mathrm{SBD}$ 基质的压电 效应. 本文首次开展了基于ML的温度传感研究, 这有望在没有激发 光源的情况下实现应力和温度的双重功能检测. 结合MOF的多功 能性和ML发射, 本研究为开发更多用途、更有趣的多功能材料提 供了独特的见解, 同时拓展了MOF和ML材料的应用范围. 